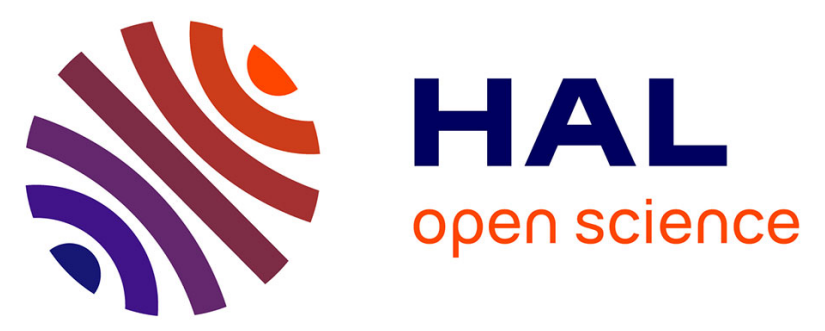

\title{
Combining magnetic nanoparticles with cell derived microvesicles for drug loading and targeting
}

Amanda K.A. Silva, Nathalie Luciani, Florence Gazeau, Kelly Aubertin, Stéphanie Bonneau, Cédric Chauvierre, Didier Letourneur, Claire Wilhelm

\section{To cite this version:}

Amanda K.A. Silva, Nathalie Luciani, Florence Gazeau, Kelly Aubertin, Stéphanie Bonneau, et al.. Combining magnetic nanoparticles with cell derived microvesicles for drug loading and targeting. Nanomedicine: Nanotechnology, Biology and Medicine, 2015, 11 (3), pp.645-655. 10.1016/j.nano.2014.11.009 . hal-01244559

\section{HAL Id: hal-01244559 \\ https://hal.science/hal-01244559}

Submitted on 13 Jun 2016

HAL is a multi-disciplinary open access archive for the deposit and dissemination of scientific research documents, whether they are published or not. The documents may come from teaching and research institutions in France or abroad, or from public or private research centers.
L'archive ouverte pluridisciplinaire HAL, est destinée au dépôt et à la diffusion de documents scientifiques de niveau recherche, publiés ou non, émanant des établissements d'enseignement et de recherche français ou étrangers, des laboratoires publics ou privés. 


\section{Accepted Manuscript}

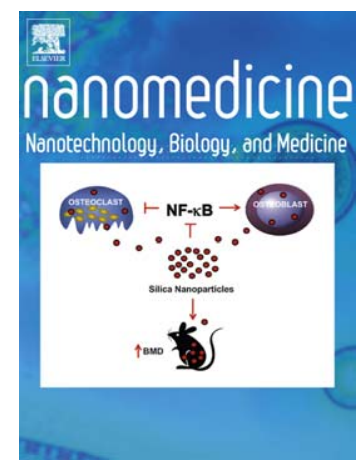

PII:

DOI:

S1549-9634(15)00003-9

Reference:

doi: $10.1016 /$ j.nano.2014.11.009

To appear in: $\quad$ Nanomedicine: Nanotechnology, Biology, and Medicine

Received date: 29 November 2013

Revised date: $\quad 31$ October 2014

Accepted date: 19 November 2014

Please cite this article as: Silva Amanda K.A., Luciani Nathalie, Gazeau Florence, Aubertin Kelly, Bonneau Stéphanie, Chauvierre Cédric, Letourneur Didier, Wilhelm Claire, Combining magnetic nanoparticles with cell derived microvesicles for drug loading and targeting, Nanomedicine: Nanotechnology, Biology, and Medicine (2015), doi: 10.1016/j.nano.2014.11.009

This is a PDF file of an unedited manuscript that has been accepted for publication. As a service to our customers we are providing this early version of the manuscript. The manuscript will undergo copyediting, typesetting, and review of the resulting proof before it is published in its final form. Please note that during the production process errors may be discovered which could affect the content, and all legal disclaimers that apply to the journal pertain. 
Combining magnetic nanoparticles with cell derived microvesicles for drug loading and targeting

Amanda K. A. Silva ${ }^{1,2}$, Nathalie Luciani ${ }^{1}$, Florence Gazeau ${ }^{1}$, Kelly Aubertin ${ }^{1}$, Stéphanie Bonneau $^{3}$, Cédric Chauvierre ${ }^{2}$, Didier Letourneur $^{2}$, Claire Wilhelm $^{1} *$

${ }^{1}$ Laboratoire Matière et Systèmes Complexes, UMR 7057, CNRS and Université Paris Diderot, 10 rue Alice Domon et Léonie Duquet, 75205 Paris cedex 13, France.

${ }^{2}$ Inserm, U1148, Cardiovascular Bio-Engineering; X. Bichat Hospital, Université Paris Diderot, 46 rue H. Huchard, F-75018, Paris, France; Université Paris 13, Sorbonne Paris Cité, F-93430, Villetaneuse, France.

${ }^{3}$ Laboratoire Jean Perrin- CNRS FRE3231, Université Pierre et Marie Curie - Paris 6, Case courrier 114, 4 place Jussieu, 75252 Paris Cedex 05, France.

*Corresponding author. Tel.: +33 1 57276203; fax: +33 1 57276211. E-mail address: claire.wilhelm@univ-paris-diderot.fr.

The authors have no conflict of interest to disclose.

Word count for the abstract: 120

Complete manuscript word count: 4,714

Number of references: 50

Number of figures and or tables: 6 


\begin{abstract}
Inspired by microvesicle-mediated intercellular communication, we propose a hybrid vector for magnetic drug delivery. It consists of macrophage-derived microvesicles engineered to enclose different therapeutic agents together with iron oxide nanoparticles. Here, we investigated in vitro how magnetic nanoparticles may influence the vector effectiveness in terms of drug uptake and targeting. Human macrophages were loaded with iron oxide nanoparticles and different therapeutic agents: doxorubicin, tissue-plasminogen activator ( $t$ PA), disulfonated tetraphenylchlorin (TPCS2a) and 5,10,15,20-tetra(mhydroxyphenyl)chlorin (mTHPC). The hybrid cell microvesicles were magnetically responsive, readily manipulated by magnetic forces and MRI-detectable. Using photosensitizer-loaded vesicles, we showed that the uptake of microvesicles by cancer cells could be kinetically modulated and spatially controlled under magnetic field and that cancer cell death was enhanced by the magnetic targeting.
\end{abstract}

Keywords: cell microvesicles, drug targeting, magnetic nanoparticles, magnetic manipulation, magnetofection. 


\section{Introduction}

One of the main issues to improve therapeutic outcome in cancer therapy as well as in the treatment of other diseases consists in limiting off-target toxicity while providing high local drug concentrations at the site of interest. Active approaches rely on drug delivery systems that enable to recognize the target and/or to induce delivery or retention at the target by stimuli. ${ }^{1,2}$ Regarding the former, specific ligands may be attached to the drug delivery surface in order to specifically bind moieties that are overexpressed or mostly present on the target. ${ }^{3,4}$ Concerning the latter, targeting may be based on the presence of specific enzymes or $\mathrm{pH}$ changes at the target site or externally-triggered by light, ultrasound or magnetic field stimuli. ${ }^{5,6}$

High magnetic susceptibility of the iron oxide core enables non-invasive manipulation of magnetic drug delivery systems by the application of a magnetic field gradient. Localized magnetically-assisted capture of magnetic drug carriers at the site of interest, termed "magnetic targeting", has been investigated in vitro, ${ }^{7}$ in preclinical ${ }^{8-11}$ and in clinical settings. ${ }^{12,13}$ While the concept of magnetic drug targeting has been proposed more than 30 years ago, ${ }^{14}$ the efficacy of magnetic drug targeting has been essentially investigated for synthetic drug delivery systems. ${ }^{15-17}$ Little attention has been focused on designing biogenic drug delivery systems enabling magnetic targeting of drugs, and more particularly applicable to hydrophilic, hydrophobic, amphoteric drugs as well as drugs with high molecular weight.

Inspired by microvesicle-mediated intercellular communication, ${ }^{18}$ we have recently proposed a naturally-derived drug delivery system. It consists of microvesicles shed by cells that are engineered to enclose exogenous cargoes. ${ }^{19,20}$ Microvesicles are originated by direct budding from the cell plasma membrane. Microvesicle release may be either constitutive or consequent to cell stimulation by physical or chemical stress. ${ }^{21}$ Shed microvesicles bring 
along membrane components and cytoplasmic contents from parent cells. Cell microvesicles are recognized as important effectors in the intercellular exchange of biological signals between not only neighboring cells but also cells at remarkable distance. ${ }^{21}$ This takes place since cell microvesicles widely circulate in the vasculature. The estimated microvesicle concentration in the peripheral blood of healthy individuals is $5-50 \mu \mathrm{g} / \mathrm{ml}$. The majority of these microvesicles are originated from blood cells, mainly platelets. The second larger population of circulating microvesicles is derived from mononuclear phagocyte cell lineage. ${ }^{22}$ Therefore, it would be reasonable to choose such cell lineage for the design of a biogenic drug delivery system. Additionally, macrophages are capable of efficiently internalizing a wide range of materials, which is an important asset for loading the exogenous drug/nanoparticle cargo before the release of such materials in the shed cell microvesicles.

Thereby, macrophage-derived microvesicles may represent a unique promise in converting an autologous intrinsically biocompatible sub-cellular entity into a drug delivery system able to carry both nanoparticles and drugs. In this regard, it would be of interest to demonstrate that cell microvesicles may be loaded with different drug molecules while simultaneously enclosing nanoparticles that enable spatially-controlled drug delivery. While the encapsulation of different drug molecules would represent a proof of versatility, the demonstration of their targeting potential would enable the drug to be concentrated at the site of interest avoiding off-target effects and reducing toxicity.

Hence, the aim of this study was to co-encapsulate drugs and magnetic nanoparticles in cellderived microvesicles and investigate how the magnetic particles may influence the performance of cell-derived hybrid microvesicles as drug delivery systems. At first, we investigated if microvesicle drug loading could be extended to different drug molecules irrespective to their molecular weight, hydrophobic, hydrophilic and amphiphilic character. Next, we showed how iron oxide nanoparticles enclosed in hybrid microvesicles could enable 
spatio-temporal microvesicle magnetic manipulation. We then focused on the photosensitizerloaded microvesicles to evidence the magnetic control of both cellular uptake and cell death. In this regard, magnetic targeting of microvesicles was analyzed at the macroscopic scale by MRI, at the cellular scale by confocal microscopy and at the subcellular level by electron microscopy.

\section{Methods}

\section{Loading THP-1 macrophages with magnetic nanoparticles and drugs}

In this study, we aimed to investigate if microvesicles secreted by macrophages could be loaded with several types of drugs. Drugs with different physico-chemical properties were chosen: doxorubicin, tissue-plasminogen activator (t-PA), disulfonated tetraphenylchlorin (TPCS2a) and 5,10,15,20-tetra(m-hydroxyphenyl)chlorin (mTHPC) (Table 1). Doxorubicin is an amphoteric drug (protonable amino group and deprotonable phenolic group) ${ }^{23}$ considered to be a molecule of intermediate solubility $(\log \mathrm{P}=0.71) .{ }^{24}$ This antitumor drug has been widely used in clinics for the treatment of a broad spectrum of cancers, although cardiomyopathy is a major adverse side effect. ${ }^{25}$ t-PA is a hydrophilic macromolecule of 527 amino acids presenting a molecular weight of $68 \mathrm{kDa} .{ }^{26}$ It is a thrombolytic agent used for the treatment of acute ischemic stroke, whose main adverse effect is hemorrhage. ${ }^{27}$ TPCS2a was selected as an amphiphilic molecule. ${ }^{28}$ It is a negatively charged photosensitizer drug with endosomal localization within cells that is investigated for photochemical internalization (PCI). ${ }^{29}$ mTHPC is a reduced porphyrin that has been investigated as a photosensitizer drug for two decades until being considered nowadays an established cancer drug on the market. It is a neutral hydrophobic molecule, as such it can cross the plasma membrane and then redistribute among the membranes of organelles in the cytoplasm. ${ }^{30,31}$ 
The magnetic iron oxide nanoparticles $\left(\gamma \mathrm{Fe}_{2} \mathrm{O}_{3}\right)$ were produced by alkaline co-precipitation of $\mathrm{FeCl}_{2}$ and $\mathrm{FeCl}_{3}$ salts, according to Massart' procedure. ${ }^{32}$ Their molarity was measured by atomic emission spectroscopy.

THP-1 cells, previously treated with phorbol 12-myristate 13-acetate (PMA) (Supplementary Figure 1), were incubated with drugs and nanoparticles (5 $\mathrm{mM}$ iron in serum-free RPMI medium supplemented with $5 \mathrm{mM}$ sodium citrate) either simultaneously (2-hour coincubation of drug and nanoparticles at $37^{\circ} \mathrm{C}$ ) or sequentially (2-hour incubation with drug followed by 2-hour incubation with nanoparticles) in order to verify if the presence of nanoparticle could change the amount of uptaken drug. Incubation was followed by 2 washings in serum-free RPMI medium and an overnight chase in order to allow nanoparticle internalization by cells. In brief, incubation step was conducted in serum-free medium, while the chase step was carried out in the presence of serum. This was followed by serum-free incubation step to trigger vesicle release, as further disclosed in the next sub-section. Drug concentration was $0.5-10 \mu \mathrm{M}$ for mTHPC (Frontier Scientific) and TPCS2a (PCI Biotech), $10 \mu \mathrm{M}$ for doxorubicin (Sigma) or $0.05 \mu \mathrm{M}$ for t-PA-FITC (Abcam).

\section{Microvesicle release by precursor cells, purification and characterization}

In order to stimulate microvesicle release, THP1-macrophages were cultured in serum-free medium for 2 days. Serum depletion induces cell stress and the subsequent release of cell microvesicles. ${ }^{33}$ Microvesicle purification from the conditioned starvation medium was performed by magnetic sorting by means of a magnet which creates a magnetic field of $\mathrm{B}=$ $650 \mathrm{mT}$, and a magnetic field gradient $\operatorname{gradB}=55 \mathrm{~T} \mathrm{~m}^{-1}$ in the volume of the syringe (Supplementary methods). ${ }^{19}$ Magnetic sorting was performed after a previous centrifugation step for eliminating apoptotic bodies and dead cells (1125 g for $10 \mathrm{~min}$ ). Microvesicles were characterized in terms of size, polydispersity index, zeta potential, drug internalization and 
magnetic targeting potential at a microscopic and macroscopic level by micromagnetophoresis and MRI, respectively (Supplementary methods).

For micromagnetophoresis, microvesicles were inserted in a glass slide/coverslip chamber featuring an integrated nickel microrod. When the microrod was magnetized by a permanent magnet, the local magnetic field generated at its extremity attracted magnetic objects toward the micromagnet, covering the tip (Supplementary methods).

\section{Microvesicle uptake}

Cancer cells were incubated with mTHPC magnetic microvesicles $(0.5 \mu \mathrm{M}$ mTHPC) for 30 minutes, 2 hours or overnight at $37^{\circ} \mathrm{C}$. Alternatively, in some experiments, cancer cells incubation with microvesicles was performed at $4{ }^{\circ} \mathrm{C}$ during 30 minutes. Confocal experiments of microvesicle uptake were carried by using $\mathrm{PKH}$-label on microvesicle membrane. In addition to that, lysosomes of recipient cancer cells were stained with Lysotracker. Further method details are provided in the supporting material.

\section{Microvesicle uptake and induced cell death under a magnetic field gradient}

The spatial modulation of drug uptake by magnetic targeting was analysed for SKOV-3, TC1 and PC3 cells. Cancer cells were seeded in culture dishes presenting a diameter of $35 \mathrm{~mm}$. Dish surface was virtually divided in three equal parts (further details are provided in the supporting material) and a magnet was placed at one of the edges. A magnetic field strength of $150 \mathrm{mT}$ was employed (rectangular magnet). Following PBS washing, the cells were incubated with $1 \mathrm{~mL}$ of magnetic mTHPC microvesicles or mTHPC in solution at $0.5 \mu \mathrm{M}$ for 2 hours in the dark at $37^{\circ} \mathrm{C}$ in a humidified $5 \% \mathrm{CO}_{2}$ atmosphere, with the magnet in position. After PBS washing, and magnet removal, culture dishes were incubated overnight with complete medium and then were analysed by confocal microscopy. Alternatively, the three surface parts were scraped individually and the collected cells of each fraction (the one near 
the magnet, the central fraction and the one far from the magnet) were separately lysed using triton X-100 at $0.3 \%$ final concentration. Drug concentration in each fraction was determined by fluorescence spectroscopy, as mTHPC is a fluorescent molecule.

Experiments on microvesicle-induced cell death under a magnetic field gradient were performed in the same conditions of uptake experiments and then cells underwent or not a laser exposure step using a $650-\mathrm{nm}$ diode laser at a light fluence of $10 \mathrm{~J}\left(100 \mathrm{~mW} / \mathrm{cm}^{2}\right.$ for 100 s). Cell death was evaluated by propidium iodide assay and MTT test. Further method details are provided in the supporting material.

\section{Results}

\section{Loading of macrophages with both drugs and magnetic nanoparticles}

We first investigated the initial uptake of drugs by macrophages after simultaneous or sequential incubation with iron oxide nanoparticles by confocal microscopy (Supplementary Figure 2). Doxorubicin, TPCS2a, and mTHPC uptake was observed by direct drug fluorescence emission. For t-PA, uptake was evaluated via the fluorescence emission of its FITC-conjugated molecule. Iron oxide nanoparticle co-incubation did not influence the uptake of doxorubicin nor t-PA. As expected, doxorubicin displayed a nuclear localization which is in agreement with its action of interfering with DNA replication via DNA intercalation. t-PA localized into the cytoplasm, as already observed for astrocytes. ${ }^{34}$

Interestingly, the uptake of TPCS2a and mTHPC was quantitatively enhanced by coincubation with nanoparticles (Supplementary Figure 3). Supplementary Figure 4 demonstrates the simultaneous internalization of photosensitizer drugs and nanoparticles. Magnetic nanoparticles were identified into endosomes/lysosomes which magnetically aligned one another in presence of a magnetic field. Red fluorescence from TPCS2a was 
observed in endosomes/lysosomes colocalizing with nanoparticles or widespread in cytoplasm due to mTHPC (Supplementary Figure 4).

\section{Production of cell-shed microvesicles loaded with the drugs and the magnetic nanoparticles}

Once macrophages were loaded with both iron oxide nanoparticles and drugs, cells were cultured for 2 days in serum-free medium in order to trigger microvesicles release. The microvesicle production method used herein was based on cell stress induced by serum deprivation. ${ }^{35}$ Such pro-apoptotic stimulus elicits phosphatidylserine translocation to the outer membrane leaflet. Microvesicles have intermediate size $(200 \mathrm{~nm}-1 \mu \mathrm{m})$ between exosomes and apoptotic bodies and result from remodeling of cell membrane and formation of outward protrusions. ${ }^{36-39}$ Serum deprivation allows triggering microvesicle shedding without any chemical or biomolecule. Therefore, no further processing is required to eliminate the vesiculation trigger agent. A centrifugation step was carried out in order to set aside the subpopulation of apoptotic bodies from microvesicle one. As a last step, cell-shed microvesicles were magnetically sorted overnight in order to select iron oxide loaded microvesicles.

\section{Microvesicle characterization}

Microvesicles presented an average hydrodynamic size of $673 \pm 168 \mathrm{~nm}$ (Supplementary Figure 5), which is in keeping with characteristic microvesicle size range from $200 \mathrm{~nm}$ to $1000 \mu \mathrm{m} .{ }^{39}$ A polydispersity index of 0.3 was obtained, which is also in agreement with natural broad size distribution of microvesicles. A zeta potential of $-30 \mathrm{mV}$ was observed, indicating a negatively charged surface.

To demonstrate the loading of drug and nanoparticles in microvesicles, a magnetophoresis technique was used to record microvesicle trajectories in the presence of a magnetic field gradient created by a micromagnet (Figure 1a). All obtained microvesicles, irrespective to the 
drug, successfully accumulated at the micromagnet tip (Figure 1b). Micromagnetophoresis set-up was also carried out under fluorescence microscopy. Images showed that the attracted microvesicles emitted the characteristic fluorescence of the drug to which precursor macrophages were incubated, indicating that the drug was encapsulated in microvesicles. The fact that microvesicles emitted drug fluorescence while presenting magnetic attraction confirmed that they were loaded with both iron oxide nanoparticles and the drug. Such observations also demonstrate the magnetic targeting potential of the produced vectors. Fluorescence emission peaks characteristic of each drug could also be detected in the lysate of purified microvesicles as observed in Supplementary Figure 6.

The magnetic targeting potential of drug-loaded magnetic microvesicles was also studied at the macroscopic level by MRI. Agarose solutions containing microvesicles were allowed to gel at $4^{\circ} \mathrm{C}$ either in the absence or in the presence of a magnet (Supplementary Figure 7) before MRI scanning. Agarose phantoms without magnet presented well-dispersed signal voids distributed uniformly throughout the sample corresponding to susceptibility artefacts created by the iron-oxide-loaded vesicles. In comparison, when gelled in the presence of the magnet, the agarose phantom displayed a hyposignal zone close to the magnet, which demonstrates magnetic attraction of the microvesicles.

In order to gain further insight into the effect of a magnetic field in the kinetics and spatiallycontrolled drug uptake and therapeutic effect, mTHPC-loaded magnetic vesicles were then chosen for the next experiments.

\section{Internalization of microvesicles by cells}

Microvesicle uptake experiments were performed with SKOV-3 human ovarian cancer cells and PC3 human prostate cancer cells. In order to visualize microvesicle internalization, microvesicle membrane was stained with a PKH membrane dye. It consists of a lipophilic 
molecule with long aliphatic tails presenting high affinity for membranes. Staining takes place by partitioning of the lipophilic dye into lipid regions of cell or vesicle membranes. Endocytosis of microvesicles was first confirmed by colocalization of PKH-staining with magnetically-responsive endosomes which were able to align in the direction of a magnetic field (Supplementary Figure 8). Additional uptake experiments, in which the lysosomes of recipient cells were labelled with Lysotracker, were performed. As presented in Figure 2 and Supplementary Figure 9, until 2 hours of incubation, green fluorescence from microvesicle membrane did not colocalize with lysosomes. This may be attributed to the delay microvesicles require reaching lysosomal compartments. Colocalization was partially evidenced after overnight incubation. In fact, green spots were observed in the same plane of lysosomes but they did not completely overlay. This may potentially result from a concurrent mechanism for entering the cell. Experiments of microvesicle internalization by cells were also carried out at $4^{\circ} \mathrm{C}$ (Supplementary Figure 10) and showed that the temperature restraint of the endocytosis pathway did not prevent microvesicles to be uptaken by cells. Indeed, it has been reported in the literature that exosomes circumvent endocytotic pathway via a direct fusion with cell membrane. ${ }^{40}$ The same may potentially apply to microvesicles.

A magnetic field gradient accelerates the transfer of the drug loaded microvesicles into cancer cells

Microvesicles were incubated with SKOV-3 cells in the presence of a magnet generating a magnetic field gradient which was constant along the cell culture surface while varying perpendicularly, as indicated in Figure 3a. mTHPC uptake kinetics mediated by free drug in solution and by mTHPC magnetic microvesicles in the presence or not of a magnetic field were quantified by means of a calibration curve (Supplementary Figure 11). At 30 minutes, the free drug in solution promoted higher drug uptake than the microvesicles. This is likely due to the fast transfer of free drugs compared to the transfer of drugs protected by 
microvesicle membrane. A such time-delayed uptake compared to the free drug in solution, has already been reported for other vectors either of synthetic or of natural origin 41,42 . However, when incubated with drug-loaded microvesicles, an almost 2-fold enhancement (P $<0.02$ ) in drug uptake could be observed at the earliest time point in the presence of the magnet. After 2 hours, drug uptake increased up to $0.8 \mathrm{fmol} /$ cell irrespective to the condition, which remained nearly the same for the following times (6h and 18h) (Figure 3b).

\section{Magnetic targeting of microvesicles to cancer cells}

Spatial targeting of microvesicles was next assessed on SKOV-3 cells incubated with PKH67stained microvesicles in the presence of a magnet generating a magnetic field gradient along the culture surface (Figure 4a). Confocal microscopy imaging revealed that fluorescence emission from PKH67-stained microvesicle membrane as well as from mTHPC drug cargo was very low in the region distal to the magnet. In contrast, the closer from the magnet, the higher the fluorescence emission from both microvesicle membrane and drug cargo (Figure 4b). Consistently, quantitative analysis from fluorescence spectroscopy of SKOV-3 cell lysate revealed a 20 -fold higher drug concentration in the magnet region compared to the spatially opposite area (Figure 4c). Due to their magnetic cargo, microvesicles experienced an increased magnetic force with decreasing distance from the permanent magnet. As a result, microvesicles were attracted to the close proximity of the magnet, thus modulating their interactions with SKOV-3 cells. Similar results were obtained with murine cervical cancer TC-1 cells (Supplementary Figure 12) and human prostate cancer PC3 cells (Supplementary Figure 13).

The magnetic cell targeting was also evaluated at the subcellular scale by TEM analysis of cancer cells after incubation with microvesicles in the presence of a magnetic field gradient. TEM micrographs revealed abundant electron-dense endosomes in the cytoplasm of cells at 
the magnet region, indicating an important uptake of iron oxide nanoparticles. Alternatively, only few electron-dense endosomes could be visualized in cells located in the region distal to the magnet (Figure 4d).

Taken together, these results highlight the remarkable effect of magnetic targeting in the spatial modulation of drug uptake in vitro.

\section{Magnetically-modulated therapeutic effect}

We then investigated if the targeting effect could induce a magnetically controlled cell death. After cancer cells were incubated with magnetic mTHPC microvesicles in the presence of the magnet, the entire plate was irradiated at $650 \mathrm{~nm}$ at a light fluence of $10 \mathrm{~J}\left(100 \mathrm{~mW} / \mathrm{cm}^{2}\right)$ during $100 \mathrm{~s}$ in order to activate the photosensitizer. Cell death was analyzed by confocal microscopy using propidium iodide staining (Figure 5a). Propidium iodide staining increased as the distance from the magnet decreased, revealing enhanced necrosis induction in the magnet region. Conversely, cells incubated to magnetic drug-free microvesicles were negative to propidium iodide staining in the magnet region after laser exposure. The same applies to non-irradiated SKOV-3 cells at the magnet, intermediate and distal regions when incubated with magnetic mTHPC microvesicles (Supplementary Figure 14). Data from MTT test (Figure 5b) was quite in agreement with propidium iodide assay. Metabolic activity was drastically reduced when cancer cells incubated with magnetic mTHPC microvesicles in the magnet region were exposed to laser while comparing to negative control. Such effect was observed in a lesser extent at the intermediate zone and no statistically significant difference was observed for the laser exposed distal region condition, the laser exposed magnetic (mTHPC-free) microvesicle condition at the magnet region or laser exposed magnet region condition when compared to negative control. 
As it can be observed, the obtained data demonstrated that cells in the magnet region exposed to laser after incubation with drug-free microvesicles presented no impact in cell viability and metabolic activity. This indicates that the magnetic microvesicle itself induced no detrimental effect. Additionally, magnetic field alone had no effect on cell viability. The obtained therapeutic effect for the condition of magnetic mTHPC microvesicles exposed to laser in the magnet region results from the magnetically induced accumulation of the vesicles in the magnet region which enhanced local drug concentration and cytotoxicity when laser exposure was applied. No significant difference was found in cell metabolic activity after cell incubation with magnetic mTHPC microvesicles without laser exposure compared to control $(\mathrm{P}=0.1154)$, as it can be observed in Supplementary Figure 15.

\section{Discussion}

In this study, we investigated cell microvesicles as biogenic vectors for magnetically targeting drugs of different molecular weight and hydrophilic/hydrophobic characters. Some authors have already succeeded in loading purified exosomes with a siRNA or anti-inflammatory drugs. ${ }^{43-46}$ For siRNA, loading was achieved by the transient disruption of exosome membrane via electroporation. ${ }^{43}$ The anti-inflammatory drug curcumin was loaded into exosomes by a different method. Due to the drug hydrophobicity, curcumin loading could be performed via hydrophobic interactions with exosomes membrane. ${ }^{44}$ Herein, we tested a different approach as the loading step was not applied directly to purified vesicles. Instead, we proposed a top-down approach in which the parent cell was loaded with both drug and magnetic nanoparticles in order to act as a fabrication unit to produce microvesicles laden with the dual cargo. The obtained results show that nanoparticles internalization in macrophages can be effective together with any of the drug tested, and that both mTHPC and 
TPCS2a molecules take advantage of nanoparticle uptake in order to further penetrate the cell, which can be beneficial for a more effective drug load into the final delivery system.

The co-encapsulation of different drugs with magnetic nanoparticles within microvesicles opens interesting perspectives. On one side, TPCS2a-loaded magnetic microvesicles would represent a smart biogenic drug vector potentially enabling both magnetic targeting and lightinduced drug release. On the other side, microvesicles loaded with doxorubicin or t-PA would be of interest for drug targeting to improve treatment efficacy while limiting the important side effects of these drugs.

The proof of magnetic targeting potential of microvesicles loaded with the tested drugs was provided by a micromagnetophoresis experiment demonstrating the attraction of the different microvesicles toward a micromagnet. Besides, magnetic nanoparticles within microvesicles could promote temporal controlled uptake of microvesicles. Magnetic field promoted faster drug uptake kinetics. These kinetic results indicate that a magnetic field influenced the kinetics of microvesicle uptake but did not increase the absolute amount of uptaken drug. The obtained results are consistent with investigations on the mechanisms of magnetofection, which consists in enhancing gene delivery by magnetic force. It has been reported that magnetic force does not induce increased endosomal uptake when the superparamagnetic gene vectors are accumulated on the cellular surface. The efficiency of magnetofection was assigned to the accelerated sedimentation of the magnetic gene vector complexes. ${ }^{47}$ Indeed, magnetofection was found to lower the required dose of vectors and to reduce incubation time, which might be important for in vivo application where the time of contact with the target cell is usually short. ${ }^{47,48}$

Besides influencing the drug uptake kinetics, the magnetic field also induced an important spatial modulation of uptake. Indeed, magnetic targeting effect was demonstrated at the 
macroscopic scale by MRI, at the cellular level by confocal microscopy and at the subcellular scale by TEM. Indeed, magnetic targeting has already been confirmed in the literature in the macroscopic scale by MRI or at the cellular level. ${ }^{16,49,50}$ However, to our knowledge, it is the first time that magnetic targeting proof is simultaneously provided at this three scale levels. Additionally, magnetic targeting was attested by investigating the spatial distribution of the components of microvesicles: membrane, drug cargo and iron oxide nanoparticles. Magnetic targeting effect was demonstrated via anisotropic drug uptake as well as anisotropic cell death. Results obtained from the spatial modulation of mTHPC uptake by magnetically guided drug targeting suggest that the magnetic cargo from the hybrid microvesicles may enhance selective drug accumulation at target site potentially implying lower therapeutic doses and reduced side effects.

In brief, we reported herein an approach to engineer microvesicles to enclose an exogenous therapeutic cargo by using parental cells loaded with magnetic nanoparticles and drugs presenting different physicochemical properties. Such bio-mimetic platform enabled drug delivery in spatio-temporal controlled manner by means of magnetic actuation, enhancing cancer cell death by magnetic targeting. Our strategy may be considered a "one for all" approach as the platform is suitable for loading different sets of drugs. Our procedure is also versatile as microvesicles could be virtually produced from a broad array of cell types. Such vectors could be therefore provided with specific endogenous targeting properties, inherited from precursor cells. Considering a product development perspective, such versatility is quite important. Additionally, microvesicles could be produced in bioreactors for scaling-up. Regarding a clinical standpoint, immune tolerance could be achieved in the case of vesicles obtained from autologous patient cells. Nevertheless, all these points have yet to be thoroughly assessed in order to foresee a clinical translation. Current and future progress in deciphering the mechanisms of microvesicle interplay with the organism may raise exciting 
new opportunities for converting cell-released vesicles into a new generation of intrinsically biocompatible drug carriers translatable to clinic.

\section{Acknowledgements}

This work was supported by the European project Magnifyco (Contract NMP4- SL-2009228622) and European Project NanoAthero (NMP4-LA-2012-309820). The authors thank Jelena Kolosnjaj-Tabi, Gwennhael Autret and the Small Animal Imaging Platform Paris Descartes PARCC-HEGP for MRI. We are grateful to Christine Longin and Sophie Chat from Mima2 platform - Inra (Jouy en Josas) for TEM analysis. The authors also thank PCI Biotech for TPCS2a. ANR (Agence Nationale de la Recherche) and CGI (Commissariat à l'Investissement d'Avenir) are gratefully acknowledged for their financial support of this work through Labex SEAM (Science and Engineering for Advanced Materials and devices) ANR 11 LABX 086, ANR 11 IDEX 05 02. We additionally thank Christine Ménager (Laboratoire PECSA-UMR 7195-CNRS-ESPCI) for providing us with the maghemite nanoparticles.

\section{References}

1. Lammers T, Kiessling F, Hennink WE and Storm G. Drug targeting to tumors: principles, pitfalls and (pre-) clinical progress. J. Control. Release 2012;161:175-187

2. Danhier F, Feron O and Préat V. To exploit the tumor microenvironment: Passive and active tumor targeting of nanocarriers for anti-cancer drug delivery. J. Control. Release 2010;148:135-146

3. Sinha R, Kim GJ, Nie S and Shin DM. Nanotechnology in cancer therapeutics: bioconjugated nanoparticles for drug delivery. Mol. Cancer Ther. 2006;5:1909-1917 
4. Pirollo KF and Chang EH. Does a targeting ligand influence nanoparticle tumor localization or uptake? Trends Biotechnol. 2008;26:552-558

5. Bae YH and Park K. Targeted drug delivery to tumors: myths, reality and possibility. J. Control. Release 2011;153:198-205

6. Owen J, Pankhurst Q and Stride E. Magnetic targeting and ultrasound mediated drug delivery: Benefits, limitations and combination. Int. J. Hyperthermia 2012;28:362-373

7. Ohulchanskyy TY, Kopwitthaya A, Jeon M, Guo M, Law W-C, Furlani EP, et al. Phospholipid micelle-based magneto-plasmonic nanoformulation for magnetic field-directed, imaging-guided photo-induced cancer therapy. Nanomedicine: NBM 2013;9:1192-1202

8. Tietze R, Lyer S, Dürr S, Struffert T, Engelhorn T, Schwarz M, et al. Efficient drugdelivery using magnetic nanoparticles - biodistribution and therapeutic effects in tumour bearing rabbits. Nanomedicine: NBM 2013;9:961-971

9. Alexiou C, Arnold W, Klein RJ, Parak FG, Hulin P, Bergemann C, et al. Locoregional Cancer Treatment with Magnetic Drug Targeting. Cancer Res. 2000;60:6641-6648

10. Chertok B, David AE and Yang VC. Polyethyleneimine-modified iron oxide nanoparticles for brain tumor drug delivery using magnetic targeting and intra-carotid administration. Biomaterials 2010;31:6317-6324

11. Arias JL, Reddy $\mathrm{LH}$ and Couvreur P. $\mathrm{Fe}_{3} \mathrm{O}_{4} /$ chitosan nanocomposite for magnetic drug targeting to cancer. J. Mater. Chem. 2012;22:7622-7632

12. Lübbe AS, Alexiou $\mathrm{C}$ and Bergemann C. Clinical applications of magnetic drug targeting. J. Surg. Res. 2001;95:200-206

13. Lübbe AS, Bergemann C, Riess H, Schriever F, Reichardt P, Possinger K, et al. Clinical experiences with magnetic drug targeting: a phase I study with 4'-epidoxorubicin in 14 patients with advanced solid tumors. Cancer Res. 1996;56:4686-4693 
14. Senyei A, Widder $\mathrm{K}$ and Czerlinski G. Magnetic guidance of drug-carrying microspheres. J. Appl. Phys. 1978;49:3578-3583

15. Veiseh O, Gunn JW and Zhang M. Design and fabrication of magnetic nanoparticles for targeted drug delivery and imaging. Adv. Drug Deliv. Rev. 2010;62:284-304

16. Fortin-Ripoche J-P, Martina MS, Gazeau F, Ménager C, Wilhelm C, Bacri J-C, et al. Magnetic Targeting of Magnetoliposomes to Solid Tumors with MR Imaging Monitoring in Mice: Feasibility1. Radiology 2006;239:415-424

17. Rivière C, Martina M-S, Tomita Y, Wilhelm C, Dinh AT, Ménager C, et al. Magnetic Targeting of Nanometric Magnetic Fluid-loaded Liposomes to Specific Brain Intravascular Areas: A Dynamic Imaging Study in Mice1. Radiology 2007;244:439-448

18. Ratajczak J, Wysoczynski M, Hayek F, Janowska-Wieczorek A and Ratajczak M. Membrane-derived microvesicles: important and underappreciated mediators of cell-to-cell communication. Leukemia 2006;20:1487-1495

19. Silva AK, Kolosnjaj-Tabi J, Bonneau S, Marangon I, Boggetto N, Aubertin K, et al. Magnetic and Photoresponsive Theranosomes: Translating Cell-Released Vesicles into Smart Nanovectors for Cancer Therapy. ACS Nano 2013;7 4954-4966

20. Silva AK, Di Corato R, Pellegrino T, Chat S, Pugliese G, Luciani N, et al. Cellderived Vesicles as a Bioplatform for the Encapsulation of Theranostic Nanomaterials. Nanoscale 2013;5:11374-11384

21. Camussi G, Deregibus MC, Bruno S, Cantaluppi V and Biancone L. Exosomes/microvesicles as a mechanism of cell-to-cell communication. Kidney Int. 2010;78:838-848

22. Hunter MP, Ismail N, Zhang X, Aguda BD, Lee EJ, Yu L, et al. Detection of microRNA expression in human peripheral blood microvesicles. PLoS One 2008;3:e3694 
23. Janes KA, Fresneau MP, Marazuela A, Fabra A and Alonso MaJ. Chitosan nanoparticles as delivery systems for doxorubicin. J. Control. Release 2001;73:255-267

24. Formariz T, Sarmento V, Silva-Junior A, Scarpa M, Santilli C and Oliveira A. Doxorubicin biocompatible $\mathrm{O} / \mathrm{W}$ microemulsion stabilized by mixed surfactant containing soya phosphatidylcholine. Colloids Surf. B Biointerfaces 2006;51:54-61

25. Singal PK and Iliskovic N. Doxorubicin-Induced Cardiomyopathy. N. Engl. J. Med. 1998;339:900-905

26. Baruah DB, Dash RN, Chaudhari $\mathrm{M}$ and Kadam S. Plasminogen activators: a comparison. Vascul. Pharmacol. 2006;44:1-9

27. Clark WM, Wissman S, Albers GW, Jhamandas JH, Madden KP and Hamilton S. Recombinant tissue-type plasminogen activator (Alteplase) for ischemic stroke 3 to 5 hours after symptom onset. JAMA 1999;282:2019-2026

28. Wang JTW, Berg K, Hogset A, Bown SG and MacRobert AJ. Photophysical and photobiological properties of a sulfonated chlorin photosensitiser TPCS2a for photochemical internalisation (PCI). Photochem. Photobiol. Sci. 2013;12:519-526

29. Berg K, Selbo PK, Prasmickaite L, Tjelle TE, Sandvig K, Moan J, et al. Photochemical Internalization A Novel Technology for Delivery of Macromolecules into Cytosol. Cancer Res. 1999;59:1180-1183

30. Mojzisova H, Bonneau S and Brault D. Structural and physico-chemical determinants of the interactions of macrocyclic photosensitizers with cells. Eur Biophys J. 2007;36:943-953 31. Senge MO and Brandt JC. Temoporfin (Foscan ${ }^{\circledR}$, 5, 10, 15, 20-Tetra (mhydroxyphenyl) chlorin)—A Second generation Photosensitizer. Photochem. Photobiol. 2011;87:1240-1296 
32. Béalle G1, Di Corato R, Kolosnjaj-Tabi J, Dupuis V, Clément O, Gazeau F, et al. Ultra magnetic liposomes for MR imaging, targeting, and hyperthermia. Langmuir 2012;28:1183411842

33. Luciani N, Wilhelm C and Gazeau F. The role of cell-released microvesicles in the intercellular transfer of magnetic nanoparticles in the monocyte/macrophage system. Biomaterials 2010;31:7061-7069

34. Cassé F, Bardou I, Danglot L, Briens A, Montagne A, Parcq J, et al. Glutamate controls tPA recycling by astrocytes, which in turn influences glutamatergic signals. $J$. Neurosci. 2012;32:5186-5199

35. Jimenez JJ, Jy W, Mauro LM, Soderland C, Horstman LL and Ahn YS. Endothelial cells release phenotypically and quantitatively distinct microparticles in activation and apoptosis. Thromb. Res. 2003;109:175-180

36. Hugel B, Martínez MC, Kunzelmann C and Freyssinet J-M. Membrane microparticles: two sides of the coin. Physiology 2005;20:22-27

37. Taylor RC, Cullen SP and Martin SJ. Apoptosis: controlled demolition at the cellular level. Nat. Rev. Mol. Cell. Biol. 2008;9:231-241

38. Muralidharan-Chari V, Clancy JW, Sedgwick A and D'Souza-Schorey C. Microvesicles: mediators of extracellular communication during cancer progression. J. Cell. Sci. 2010;123:1603-1611

39. Beyer C and Pisetsky DS. The role of microparticles in the pathogenesis of rheumatic diseases. Nat. Rev. Rheumatol. 2010;6:21-29

40. van den Boorn JG, Schlee M, Coch C and Hartmann G. SiRNA delivery with exosome nanoparticles. Nat. Biotech. 2011;29:325-326

41. Aljabali AA, Shukla S, Lomonossoff GP, Steinmetz NF and Evans DJ. CPMV-DOX Delivers. Mol Pharm. 2012;10:3-10 
42. Lee I-H, Yu MK, Kim IH, Lee J-H, Park TG and Jon S. A duplex oligodeoxynucleotide-dendrimer bioconjugate as a novel delivery vehicle for doxorubicin in in vivo cancer therapy. J. Control. Release 2011;155:88-95

43. Alvarez-Erviti L, Seow Y, Yin H, Betts C, Lakhal S and Wood MJ. Delivery of siRNA to the mouse brain by systemic injection of targeted exosomes. Nat. Biotech. 2011;29:341-345

44. Sun D, Zhuang X, Xiang X, Liu Y, Zhang S, Liu C, et al. A novel nanoparticle drug delivery system: the anti-inflammatory activity of curcumin is enhanced when encapsulated in exosomes. Mol. Ther. 2010;18:1606-1614

45. EL Andaloussi S, Lakhal S, Mäger I and Wood MJ. Exosomes for targeted siRNA delivery across biological barriers. Adv. Drug Deliv. Rev. 2013;65:391-397

46. El-Andaloussi S, Lee Y, Lakhal-Littleton S, Li J, Seow Y, Gardiner C, et al. Exosomemediated delivery of siRNA in vitro and in vivo. Nat. Protoc. 2012;7:2112-2126

47. Huth S, Lausier J, Gersting SW, Rudolph C, Plank C, Welsch U, et al. Insights into the mechanism of magnetofection using PEI-based magnetofectins for gene transfer. J. Gene Med. 2004;6:923-936

48. Scherer F, Anton M, Schillinger U, Henke J, Bergemann C, Kruger A, et al. Magnetofection: enhancing and targeting gene delivery by magnetic force in vitro and in vivo. Gene Ther. 2002;9:102-109

49. Chertok B, Moffat BA, David AE, Yu F, Bergemann C, Ross BD, et al. Iron oxide nanoparticles as a drug delivery vehicle for MRI monitored magnetic targeting of brain tumors. Biomaterials 2008;29:487-496

50. Jiang J-S, Gan Z-F, Yang Y, Du B, Qian M and Zhang P. A novel magnetic fluid based on starch-coated magnetite nanoparticles functionalized with homing peptide. $J$. Nanopart. Res. 2009;11:1321-1330 


\section{Figure/Table legends}

Table 1: Physico-chemical properties and clinical use ${ }^{24,26-28,33,34}$ of the investigated drugs.

Figure 1: (a) Micromagnetophoresis set-up featuring a glass slide/coverslip chamber to which a $50 \mu \mathrm{m}$ diameter nickel microrod was integrated. Magnetization of the nickel microrod is provided by a rectangular magnet positioned perpendicular to the micromagnet. (b) Micromagnetophoresis experiment of microvesicles obtained from THP-1 macrophages incubated $2 \mathrm{~h}$ with $5 \mathrm{mM}$ iron oxide nanoparticles and $5 \mu \mathrm{M}$ mTHPC (first line), $5 \mu \mathrm{M}$ TPCS2a (second line), $0.05 \mu \mathrm{M}$ t-PA-FITC (third line) or $10 \mu \mathrm{M}$ doxorubicin (fourth line) bright field on the left and fluorescence images on the right.

Figure 2: Confocal microscopy of SKOV-3 cells labelled with a lysosomal marker (Lysotracker in blue color) incubated with microvesicles previously stained with a membrane dye (PKH in green color) as a function of the time. Fluorescence emission from PKH-labelled vesicle membrane (first column), Lysotracker (second column), and a merge (third column) are presented.

Figure 3: (a) Representation of microvesicle-mediated uptake of mTHPC under a magnetic field. (b) mTHPC uptake as a function of the time for SKOV-3 cells incubated with mTHPC in solution or mTHPC magnetic microvesicles in the presence or not of a magnetic field ( $\mathrm{n} \geq 3$; * indicates $\mathrm{P}<0.05$ and NS means not statistically significant).

Figure 4: (a) Representation of microvesicle-mediated uptake of mTHPC under a magnetic field gradient. (b) Confocal microscopy of SKOV-3 cells after incubation with mTHPC magnetic microvesicles $(0.5 \mu \mathrm{M}$ drug load $)$ in the presence of a magnetic field gradient for 
2h: green fluorescence from microvesicle membrane (first column), red fluorescence from mTHPC (second column), blue fluorescence from Dapi-stained nuclei (third column), and merge (fourth column). (c) Quantification of the intracellular mTHPC load by fluorescence spectroscopy as a function of the distance from the magnet $(n=3)$. (d) Transmission electron microscopy of PC3 cells incubated with microvesicles in the presence of a magnetic field gradient for $2 \mathrm{~h}$. Cells presented abundant electron-dense endosomes in the magnet region (upper line) while they were scarcely observed for cells located in the region distal to the magnet (bottom line). Insets represent higher magnification views.

Figure 5: (a) Confocal microscopy of SKOV-3 cells after incubation with magnetic mTHPC microvesicles in the presence of a magnetic field gradient and laser exposure at $650 \mathrm{~nm}$ at a fluence of $10 \mathrm{~J}\left(100 \mathrm{~mW} / \mathrm{cm}^{2}\right)$ for $100 \mathrm{~s}$ : first, second and third lines. Cells incubated with magnetic (drug-free) microvesicles in the presence of a magnetic field gradient and exposed to laser are shown in the fourth row. Cells not incubated with microvesicles but in the magnet region and exposed to laser are presented in the fifth row, while cells solely exposed to laser are displayed in last row. Bright field images (first column) as well as fluorescence emission from mTHPC (second column), propidium iodide (third column), and a merge (forth column) are presented. (b) Metabolic activity obtained by MTT test for the same conditions described right above $(* * *$ indicates $\mathrm{P}<0.0001$; ** indicates $\mathrm{P}<0.01$ and NS means not statistically significant for $\mathrm{n} \geq 3$ ). 


\section{ACCEPTED MANUSCRIPT}

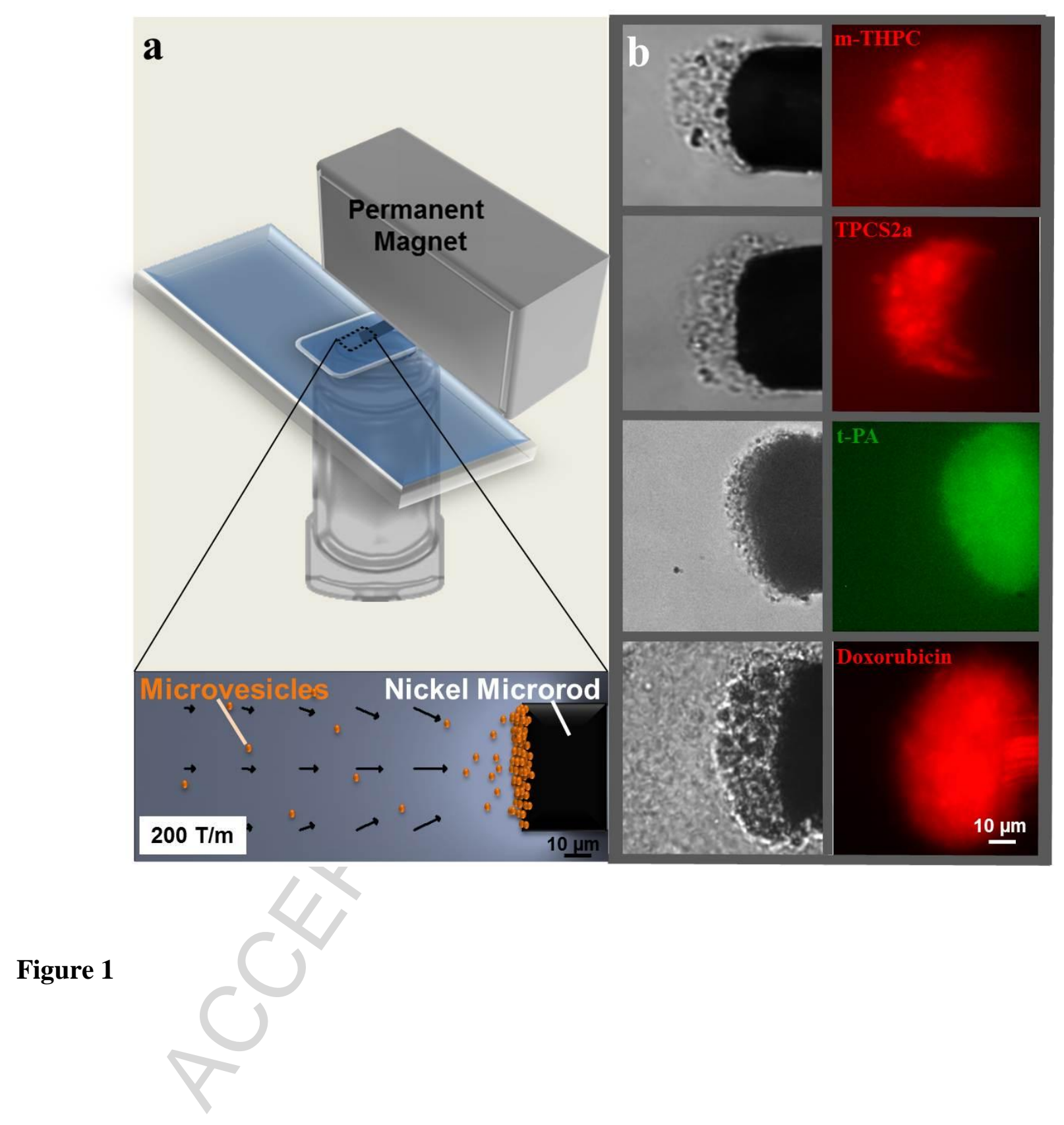




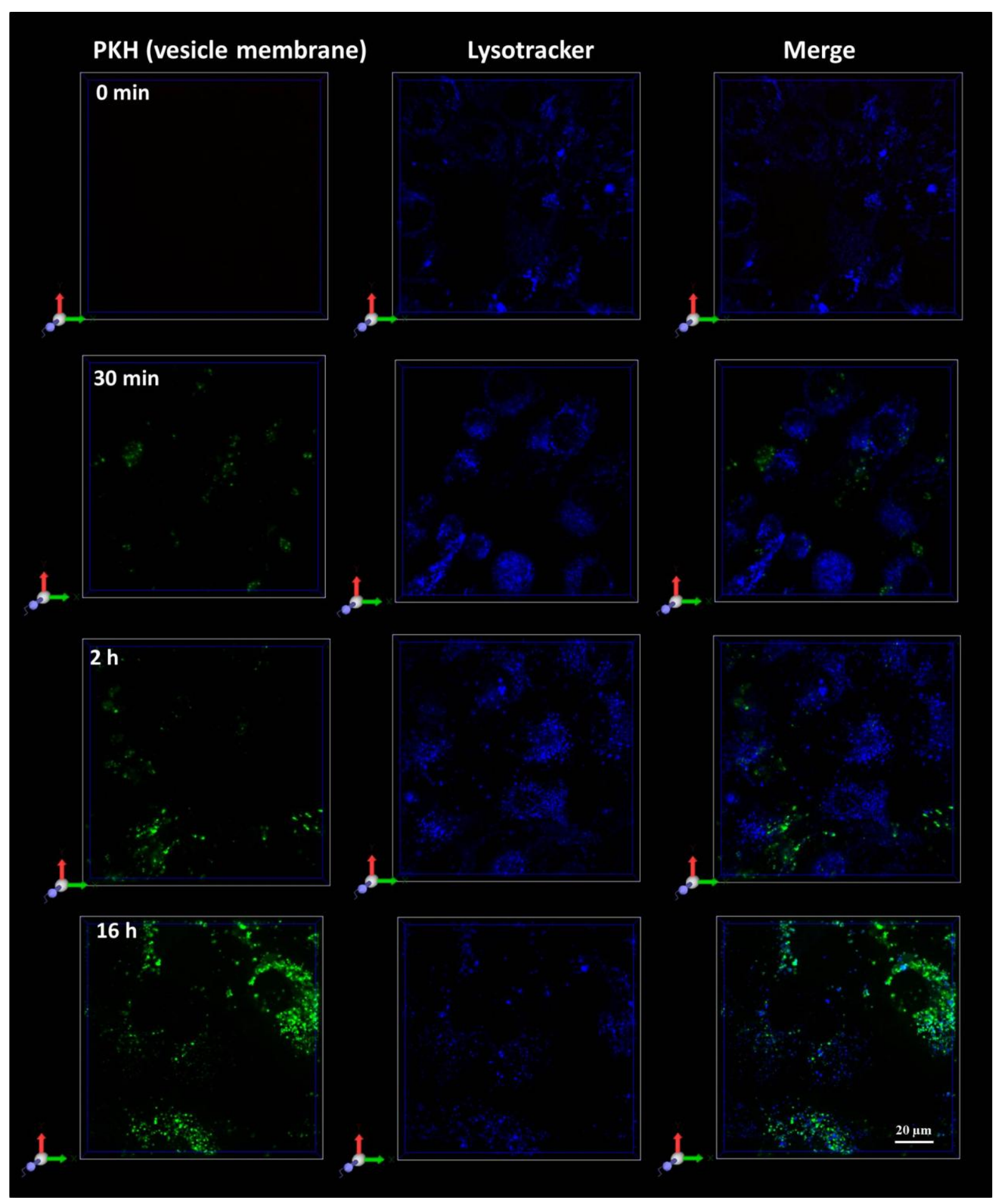

Figure 2 
a
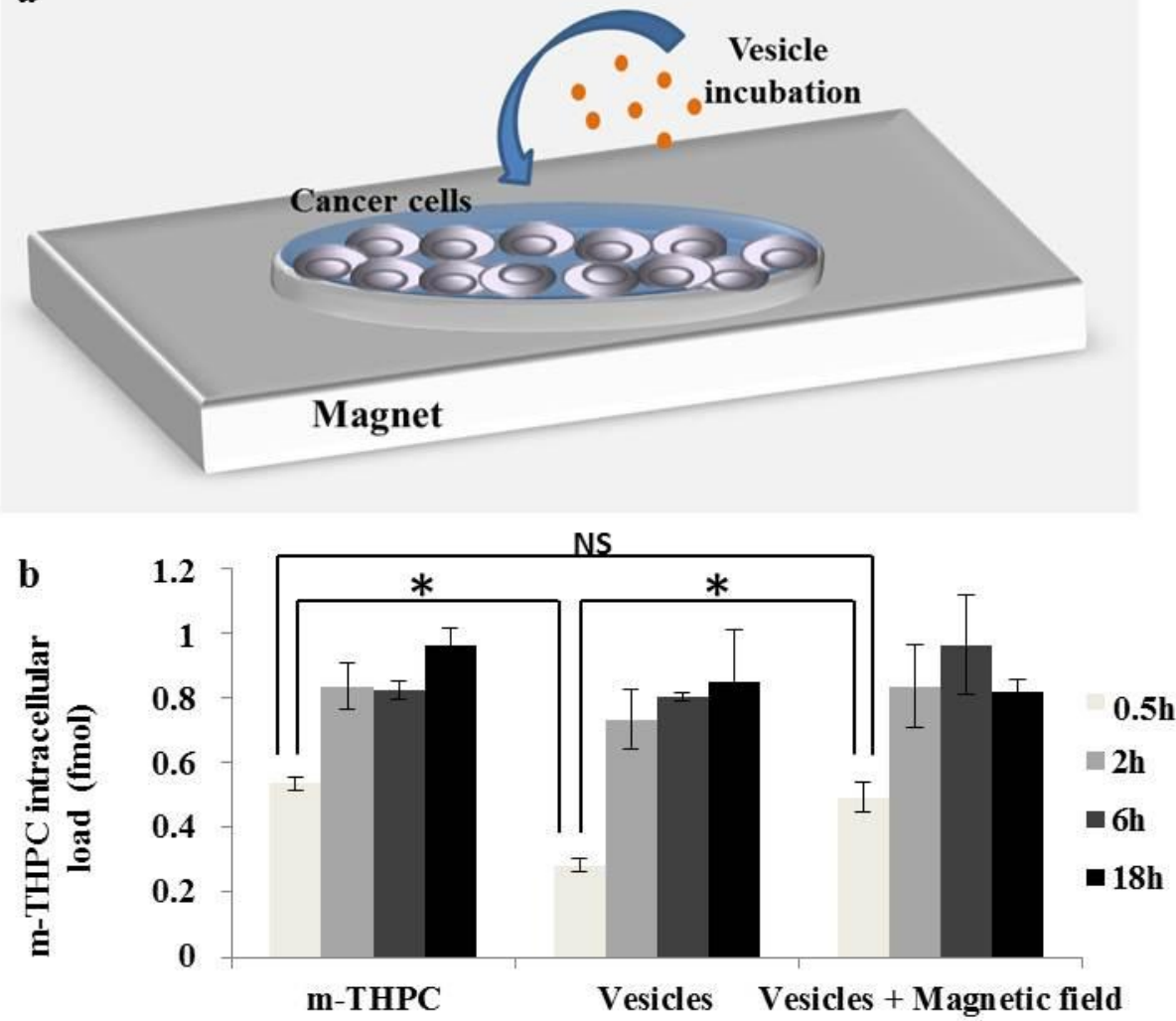

Figure 3 


\section{ACCEPTED MANUSCRIPT}

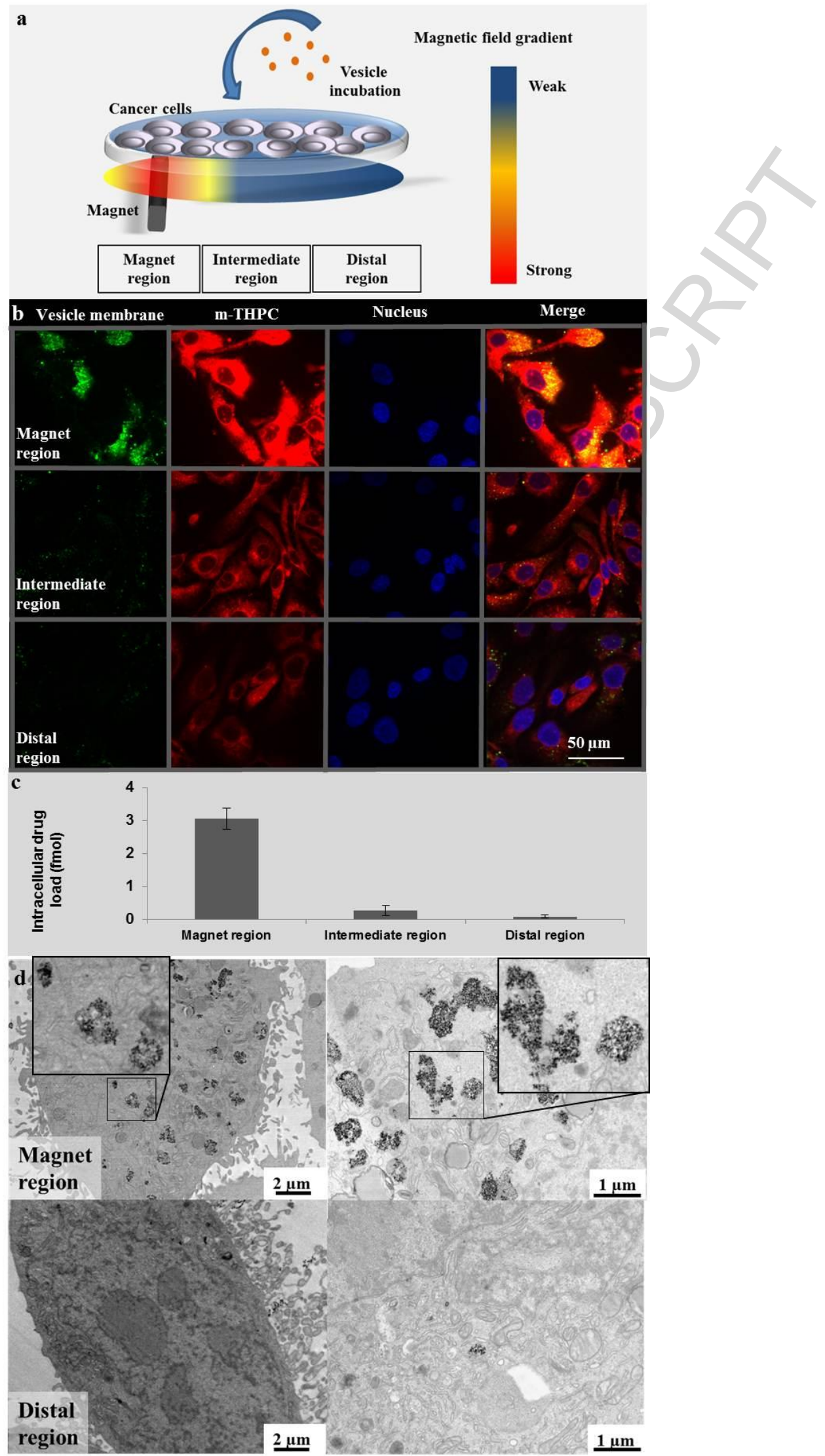

\section{Figure 4}




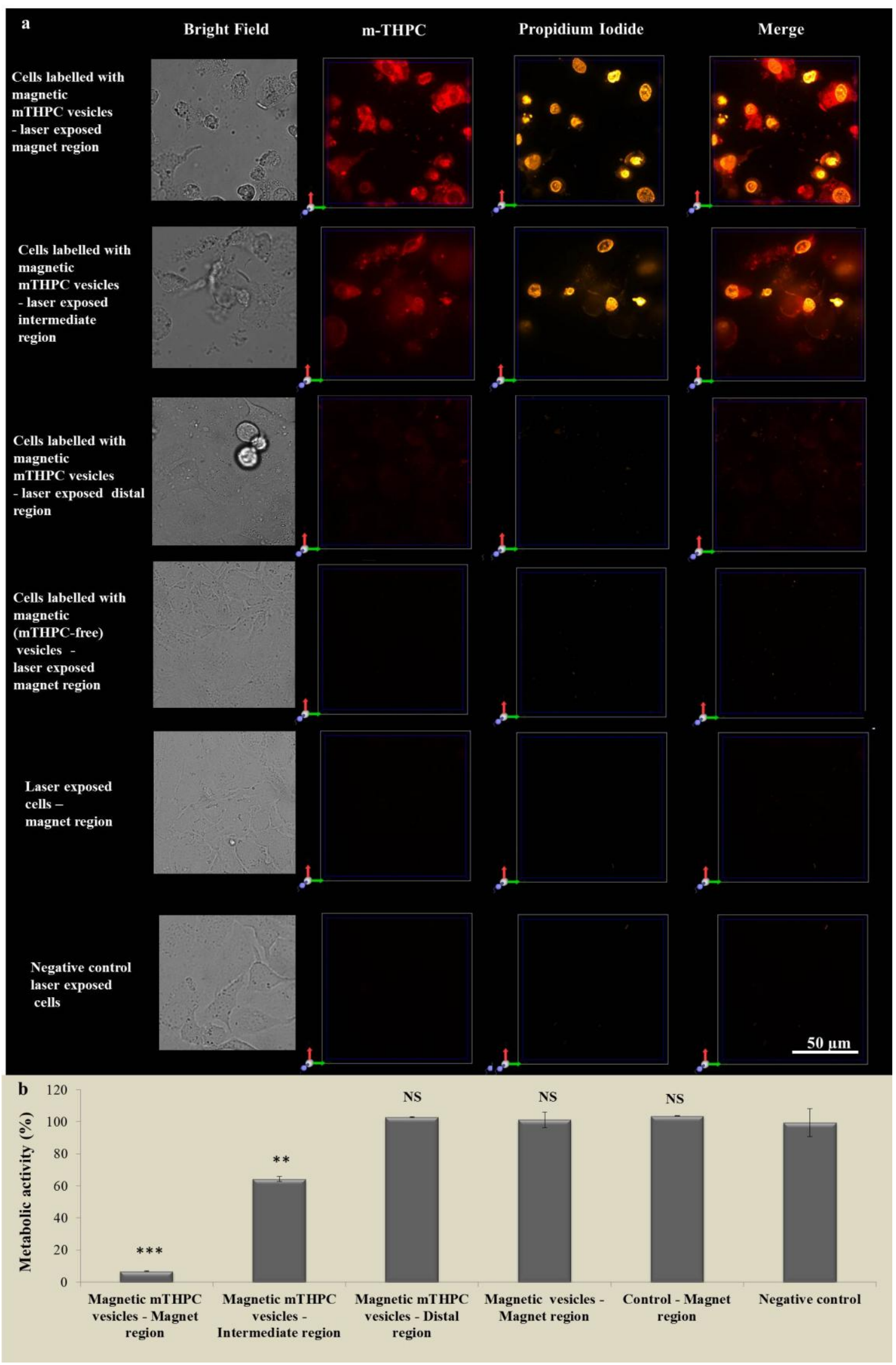

\section{Figure 5}


Table 1

\begin{tabular}{|c|c|c|c|c|}
\hline & m-THPC & TPCS2a & t-PA & Doxorubicin \\
\hline MW (Da) & 681 & 777 & 68,000 & 544 \\
\hline $\begin{array}{c}\text { Chemical } \\
\text { character }\end{array}$ & Hydrophobic & Amphiphilic & Hydrophilic & $\begin{array}{c}\text { Intermediate } \\
\text { solubility }\end{array}$ \\
\hline $\begin{array}{c}\text { Clinical } \\
\text { use }\end{array}$ & $\begin{array}{c}\text { Photosensitizer } \\
\text { anticancer drug }\end{array}$ & $\begin{array}{c}\text { Photochemical } \\
\text { internalization agent } \\
\text { under clinical trial }\end{array}$ & $\begin{array}{c}\text { Thrombolytic } \\
\text { drug }\end{array}$ & $\begin{array}{c}\text { Anticancer drug } \\
\text { (DNA intercalating) }\end{array}$ \\
\hline
\end{tabular}

Abbreviations: m-THPC: m-tetra hydroxyphenyl chlorin; TPCS2a: disulfonated tetraphenylchlorin; t-PA: tissue plasminogen activator. 


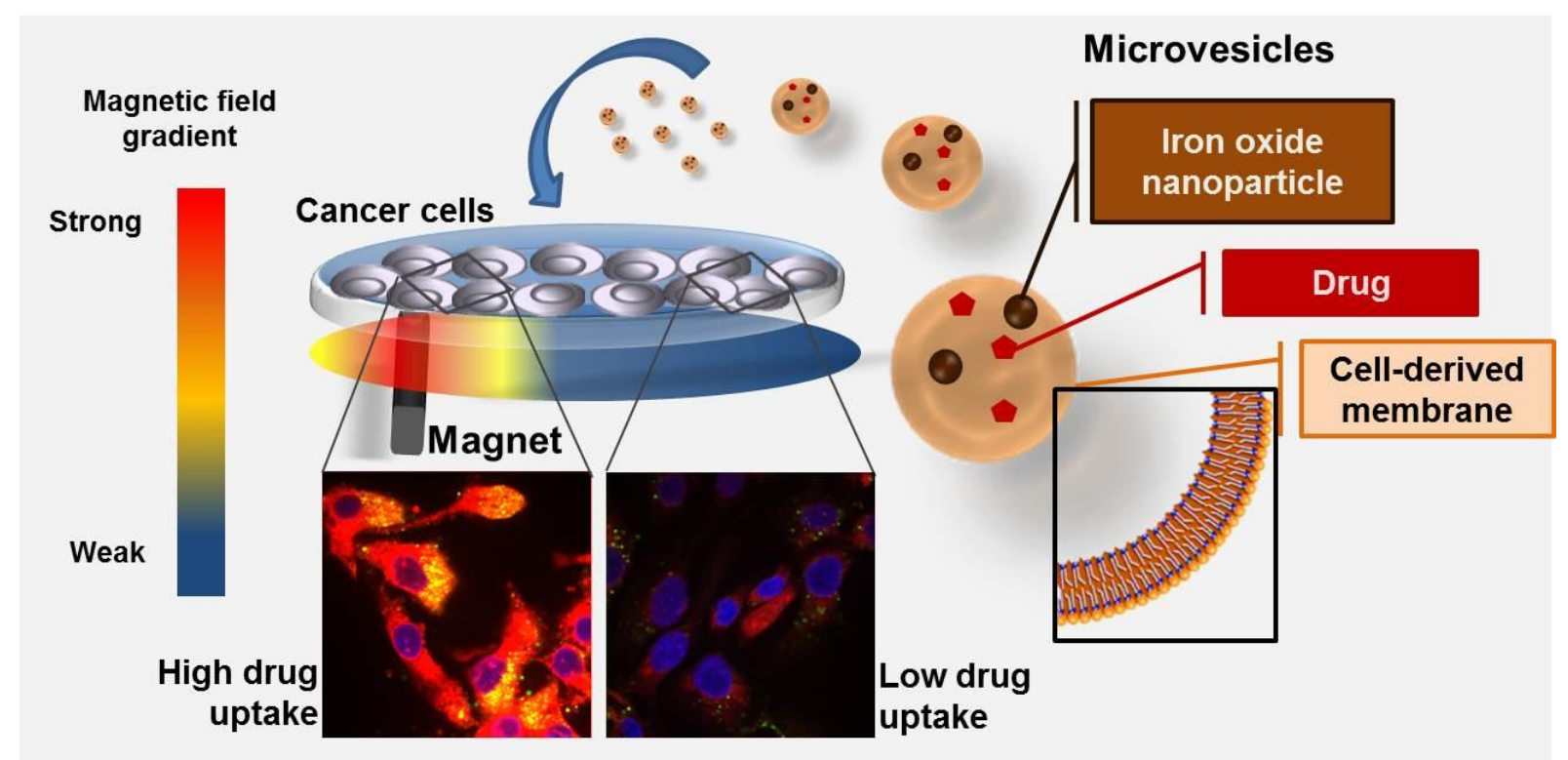

Graphical abstract text

Cell-derived microvesicles were engineered to enclose drugs and co-encapsulate magnetic nanoparticles. The uptake of microvesicles by cancer cells could be kinetically modulated and spatially controlled under magnetic field. 